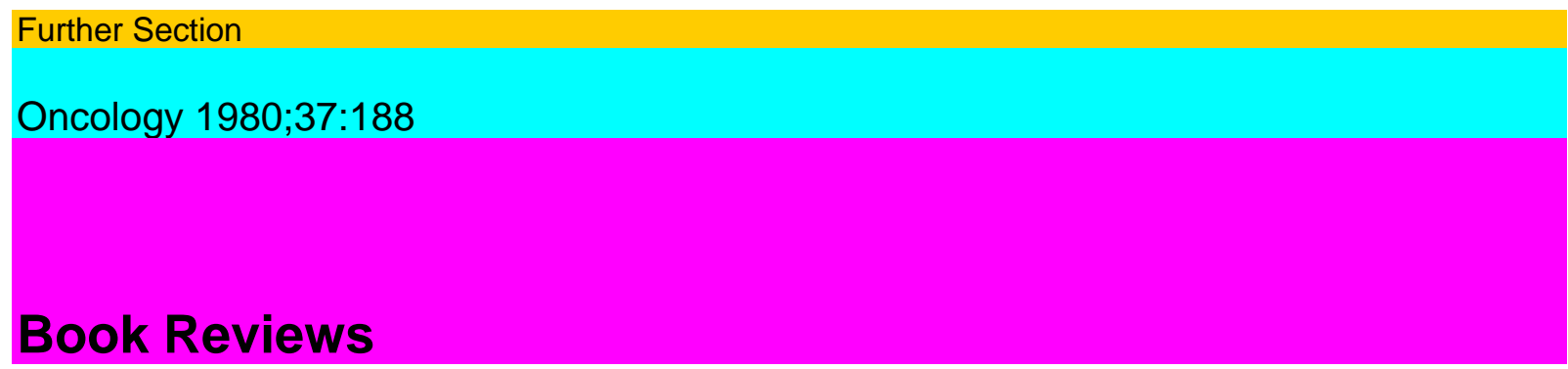

\title{
J. Staszewski
}

Epidemiology of Cancer of Selected Sites in Poland and Polish

Migrants

Ballinger, Cambridge 1976 XX + 188pp;E 12.60 ISBN 0-88410-114-2

In this book, Dr. Staszewski presents interesting information on the cancer incidence in Poland and in Polish migrants to the United States and Australia.

The baseline materials include cancer mortality data in Poland for 1959-1969 which were compared with the data on primary cancer registration for 1965-1966 in 4 selected regions of Poland. The territory, in which primary cancer registration has been conducted covers $25 \%$ of the urban and $16 \%$ of the rural population.

Cancer morbidity and mortality rates from selected tumor sites were carefully analyzed and compared with cancer morbidity and mortality data in other countries as well as with cancer mortality in Polish-born migrants to the United States and Australia.

The book contains 11 chapters including the appendices, the summary and references as well as a valuable subject index. The foreword was written by Dr. William Haenzel, Chief, Biometry Branch of the National Cancer Institute, Bethesda, Md.

The first 4 chapters include introduction, materials, methods of study and general evaluation of cancer incidence.

The author indicates that 'the purpose of this monograph is to present and analyse the occurrence of cancer of the most important primary localizations in Poland looking out for implications for cancer etiopathogenesis'.

In this respect, two main trends in epidemiological research including descriptive and analytical epidemiology were evaluated. The author stresses that the results of epidemiological studies are very important to the organization and planning of cancer control measures and to the formulation of the hypotheses on cancer etiopathogenesis.

For the comparison of the data the standardized rates, graphic method and a number of indices were widely used.

Before giving consideration to the established regularities in cancer incidence in Poland the author presented some general data about Poland on the base of geographic and demographic data.

According to Polish mortality statistics, cancer accounted for $17 \%$ of all deaths in 1969 showing an increase from $9.5 \%$ in 1959 . This increase can be ascribed to the decreasing mortality from infectious diseases, to the increase of longevity, improvement of cancer diagnosis and to a true rise in the frequency of occurrence of some types of cancer.

In comparison with other countries Poland is characterized by a relatively low cancer morbidity and mortality rates.

Mortality rates from cancer of all sites are higher for Polish males than for females. The M/F ratio increased from 1.30:1 in 1959-1961 
to $1.42: 1$ in 1967-1969. The author indicates that this can be explained by the increase in the male lung cancer incidence. Since 1959-1969 the rise in cancer mortality rates was observed both in the urban and rural populations. The highest cancer mortality rates were found among city inhibitants; however, the gradual decrease in this difference was noted particularly for older age groups.

The dynamics of cancer morbidity rates in Poland cannot be traced because the period of observation is too short for proper evaluation.

Due to the fact that cancer is not a single disease but a group of diseases the author presents the analysis of the main tumor sites. Chapters 5-10 are devoted to this problem (cancers of the stomach, intenstines, lungs, breast, uterus and prostata).

Stomach cancer is the most frequent cause of death in Poland, however, the decrease of its incidence was noted both in males and females. The incidence data from 4 regional registries rank stomach cancer first in males and second in females. Only in Warszawa does stomach cancer rank second to lung cancer in males and third after uterine cervix and breast cancer in females. The author states that in recent years the stomach and uterine cervix cancer risk have showed some decline while the increase in lung cancer risk is similar in Poland to the increase in other countries. The probability of the death from cancer of the intestines, breast, uterine body and prostata is also increasing.

The most interesting studies of Dr. Staszewski are the information about cancer mortality rates in Polish-born migrants. Thus, in 1950 stomach cancer mortality among Polish-born Americans was as high as in Poland while 10 years later it had decreased. Mortality from cancer of the breast, uterus and prostate among these Polish migrants to the United States has also increased from the low level reported for Poland, but has not yet reached the high level for natives of the host country. At the same time the author noted that lung cancer mortality among Polish-born Americans was higher than either in Poland or among native Americans. The same tendencies were revealed for Polish-born migrants to Australia.

Of great interest is chapter 11 in which the author summarized the results of studies and also discussed and evaluated the hypotheses on etiopathogenesis of cancer of the main sites as well as aimed the investigators to conduct in the future similar studies.

The determination of geographic differences and tendencies in cancer development characteristic for different peoples as well as the studies of the effects of environmental factors in hygienic and social aspects are the base without which the problem of cancer etiopathogenesis could not be solved.

Dr.S. Staszewski's book to some extent allows us to improve our knowledge in this field.

Numerous graphic pictures

perfectly performed

confirm the statements expressed by the author.

The data presented in the monograph are valuable reference material characterizing cancer occurrence in Poland. 